Article

\title{
A Framework to Evaluate the Effects of Organizational Resilience on Service Quality
}

\author{
Alessandro Annarelli ${ }^{1, *} \mathbb{( D}$, Cinzia Battistella ${ }^{2} \mathbb{D}$ and Fabio Nonino ${ }^{1}(\mathbb{D}$ \\ 1 Department of Computer, Control and Management Engineering, Sapienza University of Rome, \\ Via Ariosto 25, 00185 Rome, Italy; fabio.nonino@uniroma1.it \\ 2 Polytechnic Department of Engineering and Architecture, University of Udine, 33100 Udine, Italy; \\ cinzia.battistella@uniud.it \\ * Correspondence: alessandro.annarelli@uniroma1.it
}

Received: 5 December 2019; Accepted: 26 January 2020; Published: 28 January 2020

\begin{abstract}
Disruptions and unexpected turbulences characterize the contemporary service industry and pose greater criticalities to organizations that have concerns about both their survival and their business sustainability. The answer to this challenge is to design an organizational system aimed at enhancing so-called organizational resilience. The aim of this work is to present and test an assessment methodology based on a framework that links key static and dynamic characteristics of firms as resilient systems and relates these characteristics with service quality preservation after disruptions. The framework adopts a set of indicators, namely resilience dimensions, to comprehend the real effects of resilience characteristics with service quality dimensions. Through the analysis of a real case, we provided evidence of how the model can be applied in a backward perspective to understand fault modes of specific events. Results show how inadequacies in terms of resilience characteristics determine losses of service quality, and also how the model can be used as a predictive tool to determine in which area companies should intervene in order to improve resilience and service quality.
\end{abstract}

Keywords: organizational resilience; resilient systems; resilience characteristics; resilience dimensions; service dimensions

\section{Introduction}

Nowadays, the competitive environment always poses new threats and challenges to firms due to an increased turbulence in markets and industries. In addition, the natural and social environment in which companies operate poses a whole series of challenges and threats that can seriously jeopardize the business operation and continuity: this is the case of natural disasters, terrorism, floods, diseases, economic crises, and recession, as well as mass failures, and breakdowns and malfunctions of physical and technological systems [1,2].

In this challenging context, companies need to be resilient. Resilience, originally referred to "jumping back" (original meaning from the Latin resiliere) and to the ability of recovery, emerged in academic literature thanks to the work of Holling [3] in the field of ecology and ecosystems. Then resilience studies spread to a multitude of research fields, as a general property of many different systems, intended as the ability of resisting to disturbances and recovery [4-9].

For what concerns organizational resilience, we can define as "the organization's ability to face disruptions and unexpected events thanks to the strategic awareness and a linked operational management of internal and external shocks" [10]. Furthermore, it is important to highlight two particular attributes of resilience coming from its static and dynamic nature. Static resilience is mostly based on preparedness and preventive measures to minimize threats in terms of probability of 
occurrence and potential impact, while dynamic resilience is more focused on the effective management of accidents and unforeseen events to shorten unfavorable aftermaths and maximize the organization's speed of recovery [11,12].

Therefore, it is vital for companies to put in action measures for building organizational resilience to maintain competitiveness and to secure business sustainability. To this extent, the aim of this work is to propose a methodology to evaluate the effectiveness of organizational resilience strategy and related actions in the specific context of the service industry. In the general context described above, companies are facing a series of new threats related to cyber-attacks becoming always more relevant and related to the key topics of cyber security and digital transformation [13]. In the past, literature on organizational resilience focused mostly on product companies and supply chains; however, there is a need to investigate in greater detail the development of resilience in service companies [14]. According to the authors' knowledge, there is a gap in the literature that concerns the development of a set of indicators to evaluate resilience measures put in action in the specific context of service companies. Nowadays, service companies are facing the same types of problems. Building resilience, while maintaining competitiveness and stability of business operations, is a key challenge, and our aim is therefore proposing and testing a framework that makes explicit linkages between resilience characteristics and service quality preservation after a disruption.

The paper is organized as follows. Section 2 presents the theoretical background of the study. Section 3 describes the framework of analysis and Section 4 presents the case analyzed to test the applicability of the model. Section 5 contains concluding remarks together with limitations and future research directions.

\section{Theoretical Background}

\subsection{Characteristics of Resilient Systems}

Resilience is an intrinsic attribute of systems, and therefore, of companies, nurtured and fostered by developing a relevant organizational culture, which in turn, also determines the key characteristics of resilient systems, detailed below. As already reported, it is important to distinguish between elements that characterize a static or a dynamic approach to [10-12].

Moving from the literature, we derived seven elements that characterize a static approach (based on preparation and prevention) and the structural capabilities predicting a resilient organization, namely, (1) continuous monitoring, (2) anticipation ability, (3) redundancy, (4) simulation, (5) initial vulnerability, (6) focus on minor aspects, and (7) learning from mistakes. At the same time, we identified two key elements that characterize a dynamic approach (focused on timely management of crisis) toward resilience, which are (1) internal communication and (2) improvisational capabilities.

Indeed, as regards to static capabilities for resilience, organizations need to put in action continuous monitoring efforts, i.e., implementing a set of activities aimed at helping actors to constantly monitor organizational processes and eventually correct and reiterate, through information gathered, the actions taken [15]. Also, according to Weick and Sutcliffe [15], when talking about anticipation ability, we refer to the ability of foreseeing an uncontrolled undesired effect, based on small differences. Following this, another key element of resilient systems is redundancy: this term refers to the duplication of the critical components of a system with the intention of increasing its reliability, in particular for functions of vital importance to guarantee, for instance, the safety of people and plants or the continuity of production. This can be pursued by ensuring the existence of different means and actors that can perform a specific task arranged in such a way a system failure can occur only as a consequence of the simultaneous failure of all these means and actors [14]. Another two elements to be taken into account are simulation and initial vulnerability: the first one relates to the need for companies to conduct simulations and adopt simulation tools in order to be able to predict possible future scenarios and to assess the effectiveness of proposed approaches [16,17]; at the same time, in order to implement measures for resilience, organizations need to be aware of their potential vulnerabilities, defined as 
the state of being exposed to the possibility of being attacked or harmed [18]. Together with the underestimation of information sharing and communication, organizations tend to ignore minor events and/or aspects that could act as warning signals. According to Choo [19], "warning signals and events are discounted because of values, norms, and priorities that influence the evaluation and interpretation of information, so that no corrective action is taken" and that is why focus on minor aspects should play an important role in building a culture for resilience [20]. Finally, learning from mistakes is related to the ability of translating lessons learned in a new and adequate operational capacity with a particular reference to mistaken assumptions and actions.

As regards to dynamic capabilities for resilience, internal communication has always played a key role in organizations under different lights, e.g., by impacting strategic decision making and strategic planning [21,22], organizational change [23], and also human resources management by ensuring branding and employee engagement [24-26]. Accordingly, we consider internal communication as a key characteristic for enabling organizational resilience, since its ability to quickly spread information within companies goes in pair with the ability to respond timely, react, and reconfigure after unexpected events and accidents. Therefore, internal communication can be the answer to one of the major problems that can usually undermine organizational resilience, i.e., bureaucratic culture and information dispersion $[19,20,27,28]$. Improvisational capabilities are defined as "the ability to spontaneously reconfigure existing resources to build new operational capabilities to address urgent, unpredictable, and novel environmental situations" [29]. These capabilities refer to the need for companies to cope with turbulent and unpredictable environments, a characteristic that has been widely acknowledged in the literature as a key requisite for resilience [30].

\subsection{Resilience Dimensions}

Together with static and dynamic elements of resilient systems, in order to provide a complete and more immediate qualitative view of organizational resilience, there is a need to define a series of direct measures on the level of organizational resilience. Past works in the literature extensively discussed the issue of resilience dimensions. For instance, Bruneau et al. [31] presented four resilience properties, upon which depend four dimensions: the properties of robustness, redundancy, resourcefulness, and rapidity. Robustness is defined as the ability to withstand a certain level of stress without suffering loss of performance and/or function; redundancy refers to the extent to which elements of a system are substitutable or replicable; resourcefulness is intended as the ability to mobilize resources and assets to face unexpected events and/or threats; while rapidity concerns "the capacity to meet priorities and achieve goals in a timely manner to contain losses and avoid future disruption" ([31], p. 6). Furthermore, Bruneau et al. [31] identified four different dimensions that characterize resilience: technical, social, economic and organizational.

The above model has been reprised and expanded by Fischer et al. [32] who also included the aspects of vulnerability and susceptibility to assess the degree of a system's resilience: more specifically, susceptibility refers to the frequency of adverse events combined with the exposition of systems to these events.

Therefore, moving from the analysis of previous contributions, we could identify seven different areas of resilience evaluation:

1. Adaptability is the ability to adapt to changes, more specifically it has been defined by Stevenson and Spring [33] as the adaptive ability of a system in response to changes in its environment.

2. Reliability is generally defined as the probability that a system fulfils its function satisfactorily, for a specified time interval and under certain operating conditions [15].

3. Agility, defined as the ability of a system to answer and react to changes in uncertain and changing environments [34].

4. Effectiveness of resilience actions that according to the Cambridge Dictionary, is the quality of being successful in achieving what is wanted and the ability to produce the intended results. 
5. Flexibility refers to the extent and pace to which organizations adapt to sudden and radical changes, and is based on the chance of quickly interchanging elements to perform different tasks without having to reorganize all of the system's processes [15].

6. Recovery level can be expressed as the difference between the system's initial state before the malfunctioning/accident and the restore point, and it can be also compared to a desired recovery level in order to assess the performance of specific actions put in place [35,36].

7. Recovery time indicates the time needed by the system and organization to restore its normal state $[35,36]$.

\subsection{Service Dimensions}

The methodology proposed takes into account the effects of resilience on the quality of services offered by organizations. Therefore, it is crucial to define a series of dimensions and related indicators that allow evaluating the quality of services. To provide a comprehensive evaluation of service provision process, we decided to adopt a subset of service evaluation model proposed by Parasuraman et al. $[37,38]$, based on the concept of perceived quality, defined as the judgement of consumers about the overall excellence of an [39]. Linked to this concept, there are four key aspects to be considered, which are the comparison of perceived quality versus objective quality [40,41]; the concept of quality as attitude [42]; the conceptual gap between quality and satisfaction [37,43], since consumers' satisfaction can be achieved even if the perceived quality is low; and the difference between expectations compared to perceptions that represent a direct measure of the perceived quality notion $[37,44,45]$.

Following the above points/reflections, the proposed model presents a subset of six dimensions that reflect the overall quality of services, which are [37]:

- Competence: possession of the required skills and knowledge to deliver a service.

- Access: includes approachability and ease of contact.

- Reliability: refers to the consistency of performance.

- Responsiveness: the willingness and readiness in providing services.

- Credibility: it is a measure of trustworthiness, believability, and honesty.

- Security: freedom from risk and/or danger.

\section{Framework of Analysis}

Figure 1 presents the conceptual framework that summarizes relationships among variables considered in the study.

The main concept behind this framework is that companies make efforts to build an organizational culture and to put actions in practice to become resilient systems (box on the left). The effects of such actions can be measured and expressed by resilience indicators (central box) that have the purpose of interpreting the effects in seven resilience dimensions, which have an impact on maintaining service quality after a disruption (box on the right).

Logically, not all the elements of a resilient system affect an index of resilience, just as not all resilience indices affect a service feature. The following Table 1 summarizes the relationship between resilience characteristics and resilience dimensions, as emerging from the literature. 


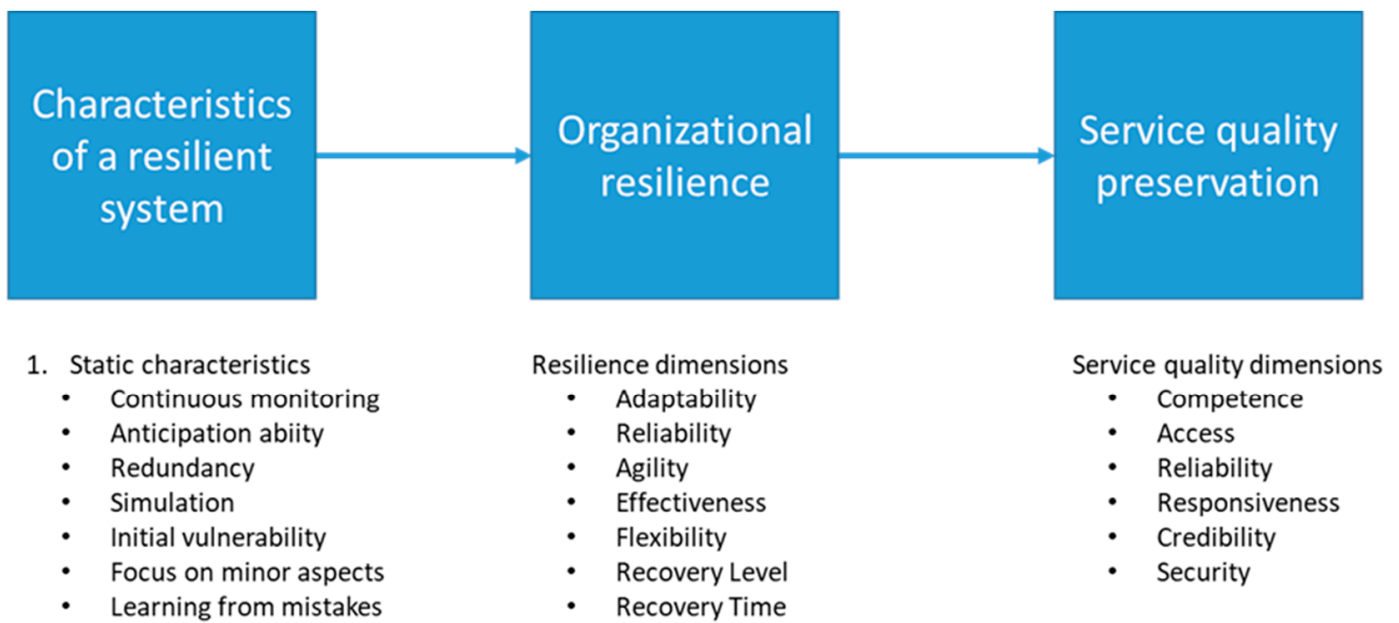

2. Dynamic characteristics

- Internal communication

- Improvisational capabilities

Figure 1. Impact of organizational resilience on service quality preservation.

Table 1. Influence of the Elements of Resilient Systems on Resilience Indexes.

\begin{tabular}{|c|c|c|c|c|c|c|c|c|}
\hline & & \multicolumn{7}{|c|}{ Resilience Dimensions } \\
\hline & & Adaptability & Reliability & Agility & Effectiveness & Flexibility & $\begin{array}{c}\text { Recovery } \\
\text { Level }\end{array}$ & $\begin{array}{c}\text { Recovery } \\
\text { Time }\end{array}$ \\
\hline \multirow{6}{*}{$\begin{array}{c}\text { Resilience } \\
\text { characteristics }\end{array}$} & $\begin{array}{l}\text { Learning from } \\
\text { mistakes }\end{array}$ & $x$ & $x$ & $x$ & $x$ & $X$ & $x$ & $x$ \\
\hline & $\begin{array}{c}\text { Internal } \\
\text { communication }\end{array}$ & $x$ & $x$ & & $x$ & $x$ & $x$ & $x$ \\
\hline & $\begin{array}{c}\text { Improvisational } \\
\text { capabilities }\end{array}$ & $x$ & & $x$ & $x$ & & $x$ & $X$ \\
\hline & $\begin{array}{l}\text { Continuous } \\
\text { monitoring }\end{array}$ & $x$ & $x$ & $x$ & $x$ & $x$ & $x$ & \\
\hline & Redundancy & & $x$ & $x$ & $x$ & & $x$ & $x$ \\
\hline & Simulation & $x$ & $x$ & $x$ & $x$ & & $x$ & \\
\hline
\end{tabular}

The Table highlights how some elements of resilience found in the literature are more influential than others on resilience indexes. Indeed, learning from mistakes affects all seven indexes, and this happens because an organization, in order to be resilient and to survive a malfunction or a crisis, must be able to draw as much knowledge as possible from mistakes made. This mechanism helps the organization to face new problems with greater ease or at least having behind it experiences to refer to as a support to decision making.

The anticipation ability affects fewer indexes, but this does not mean it is less important. Knowing how to anticipate a possible problem is not an easy task, as in most cases, problems are unexpected and there are no elements that can lead us to foresee them.

On the other hand, an important step toward resilience is acknowledging alarms and revelatory signals, and attributing them the right importance. For this reason, it is of fundamental importance for an organization to focus on minor aspects, which can hide greater problems and incidents. The role of human resources goes hand in hand with the last element considered. In particular, any employee who becomes aware of a problem or identifies the first symptoms of malfunctions should communicate this situation without any reservations. The ability to communicate these problems lies at the base of a mindful organization. To this extent, an efficient and reliable internal communication system is confirmed as a key element of a resilient organization, so that information, provisions, and problems 
can be communicated quickly, as well as decisions, in order to stem in advance, or in any case with a certain promptness, any problem that might happen.

Nonetheless, the role and relevance of improvisational capabilities can be crucial when ordinary systems might fail, or communications might not flow as planned. Indeed, investing in the development of these capabilities is essential for companies, since they ensure adaptability, flexibility, and agility, while at the same time allowing companies a certain recovery level and time.

Within a resilient organization, it certainly cannot lack a continuous monitoring system that allows not only to assess the gap between what is planned and what is actually being achieved, but also to highlight any anomaly that may arise and emerge in a system.

Redundancy is based on the concept of having more than one resource/component of the same type that can perform the same task at disposal. This helps an organization in being resilient, since it allows the replacement of defective components without severe interruptions and breakdowns. On the other hand, problems might arise when malfunctions concern integrated systems, making redundancy almost useless. To this extent, companies should not underestimate possible threats, and should avoid "feeling safe" because of redundant resources. However, advantages of redundancy are undisputable, and so is its ability in increasing organizational resilience, especially in the face of standard failures.

The simulation of scenarios is a tool of vital importance for an organization that brings radical innovations in a system, since it allows making more accurate forecasts and plans based on the results obtained. However, these simulation processes represent additional costs for the organization and it might may result in the simulations that are carried out suffering from specific environmental conditions/assumptions that can invalidate the results.

Concluding, all the features above should be considered in the process of developing resilience within an organization. A key step is fostering change within the organizational culture in order to build commitment towards resilience. Furthermore, companies must also invest in tools and mechanisms that promote resilience. This is also the case, for instance, for redundancy, a key component within an area of fundamental importance, as well as for simulations and tests.

Looking at the second part of the framework proposed, the following Table 2 reports the relationship between resilience and service quality dimensions.

Table 2. Relationship between resilience and service quality dimensions.

\begin{tabular}{cccccccc}
\hline & \multicolumn{5}{c}{ Service Dimensions } \\
\cline { 3 - 7 } & & Competence & Access & Reliability & Responsiveness & Credibility & Security \\
\hline & Adaptability & & $X$ & $X$ & $X$ & $X$ \\
Resilience & Agiliability & $X$ & $X$ & $X$ & $X$ & $X$ & $X$ \\
dimensions & Effectiveness & $X$ & $X$ & $X$ & $X$ & $X$ & $X$ \\
& Flexibility & $X$ & & $X$ & $X$ & $X$ & $X$ \\
& Recovery Level & & & & $X$ & $X$ & $X$ \\
& Recovery Time & & $X$ & $X$ & $X$ & $X$ & \\
\hline
\end{tabular}

As it can be seen from the table, the reliability of the system affects all the six dimensions of service and therefore is of vital importance for organizations aiming toward resilience. As a matter of fact, an organization that lacks reliability creates problems of access to services, creating differences between what is being offered and what is presented, and problems regarding the organization's responsiveness, as well as its competence to provide services. In addition, credibility and security are closely linked to reliability, since a lack in this aspect can result in a risk for the final customers and cause doubts.

Adaptability is a key requisite for building resilience and ensuring service quality, since knowing how to cope with environmental changes is very important for organizations as it should be able to maintain operations in almost all conditions, as well as being able to respond to changes in an uncertain and changing environment. 
Another important feature for an organization is effectiveness. In Table 1, it clearly emerged how effectiveness is related to all elements of resilient systems, and looking at Table 2, we can see how effectiveness affects almost all the service dimensions, and therefore we can state that for organizations, it is essential to be competent and prepared in carrying out tasks and reaching goals. Nevertheless, flexibility has some impact on service dimensions, even if its importance should be understood in comparison and interaction with other indicators, such as adaptability and responsiveness, as discussed above.

The recovery level and recovery time are two very important parameters that certify the quality of an organization. We can state that, when facing a problem, the more a company can recover (level) within the minimum time, the less the service quality evaluation will be affected by it.

\section{Results from Model Application}

\subsection{Case Analysis}

The case presented concerns a large European service company that went through a considerable effort of digital transformation in the last year. This process brought the company to provide itself with real-time monitoring and control infrastructure to effectively manage its service provision in a continuous way, so as to be able to proactively adapt to situations of contingent difficulties and complex events. To cope with unexpected events, the central control system was coupled with a control room that allows the verification, in real time, of the regular ongoing activities and processes.

This renovation process continued on expanding the document management services, which guarantees, for example, the management of the documentation needed for sales automation processes and contracting processes. Furthermore, the company created a multi-channel platform on which to convey all the organization's distribution channels.

Nonetheless, the company faced a serious malfunctioning that undermined the functionality of the overall infrastructure, causing non-negligible problems and losses of service. At the origin of this malfunctioning, there were some anomalies in the central system that manages all the services provided, which then propagated to almost all terminals of the company. Among the main causes, the company acknowledged a serious system bug caused by an update, together with inefficiencies and inadequacies of their platforms and infrastructure. However, this unforeseen event caused several days of malfunctioning and shut-downs to hundreds of the company's front offices.

Although the company shared emergency indications and procedures with its peripheral offices and business units, these could not manage to restore operations at an acceptable level without the intervention of a central crisis unit, and many times, interventions required a complete closure and shutdown of the systems. To restore the situation, actions undertaken concerned a full restart of the information systems and applications, inhibition of some critical functionalities, and a reset of configuration parameters when needed, but real corrective actions to the problem started only after the third day of malfunctioning.

The management of this critical event was carried out through a series of articulated actions, which included: constant monitoring support, development of specific procedures to assist peripheral offices and guarantees of continuity of service provision, extension of opening hours, and the establishment of a task force dedicated to handling the problem. Furthermore, technical solutions undertaken required $100 \%$ of available IT resources, plus the use of secondary emergency systems. Obviously, these malfunctioning and IT problems impacted severely on the quality of service provided, resulting in a lack of trust from clients and a serious reputational loss for the company. We gathered data from different sources in order to be able to analyze the six dimensions of service evaluation of the model proposed. To analyze performance in terms of competence, access, and reliability, we analyzed data from around 20,000 customers' opinions (over almost 1,500,000 customers during malfunctioning days) from the company's support website. To evaluate responsiveness, we could analyze data provided by the company. The average waiting time was evaluated with reference to a day without malfunctions 
in the critical period. The last two aspects of credibility and security were evaluated by analyzing content from Google News. The analysis was carried out by cataloguing all the articles published on the internet that contained the name of the organization combined with other keywords, such as the technical assistance company, inefficiencies, problems, inconveniences, closure, reimbursement, quality, and trust. Therefore, what emerged for the six areas was:

- Competence: what emerged from data is that almost $6 \%$ of customers claimed a lack of professionalism and professional capacity shown by employees, with an increase of $13 \%$ of unsatisfied customers compared to normal functioning periods.

- Access: more than $20 \%$ of customers spoke negatively about this point, manifesting discomfort due to excessive waiting times, with an increase of $63 \%$ of unsatisfied clients when compared to normal situations.

- Reliability: $2.5 \%$ of customers declared that services were not provided at all, while another $5.5 \%$ declared to be disappointed with the low level of service provided by the company, showing an increase of $46 \%$.

- Responsiveness: throughout malfunctioning periods, the average waiting time exhibited an increase of $222 \%$, to reduce to $58 \%$ in the first days following the incident.

- Credibility: from the analysis of contents, emerged an increase in the number of articles concerning the company by almost $300 \%$, with an increase of negative comments about the company of $1665 \%$; furthermore, $55 \%$ of articles attributed the responsibility for malfunctioning directly to the company, while the remaining $45 \%$ blamed the technical assistance company.

- Security: similarly, the increase of negative comments and opinions concerning the security provided the company raised by $818 \%$ during the malfunctioning period.

The Table 3 below summarizes the severity of the malfunctioning from service evaluation.

Table 3. Impact of malfunctioning on six dimensions of service quality.

\begin{tabular}{|c|c|c|c|c|}
\hline Dimension & Indicators & Measure & Variation & Impact Severity \\
\hline Competence & $\begin{array}{l}\text { Professionalism of } \\
\text { employees }\end{array}$ & $\begin{array}{l}\text { No. negative } \\
\text { evaluations/No. of } \\
\text { responding clients }\end{array}$ & $+13 \%$ & Low \\
\hline Access & $\begin{array}{l}\text { Perception of waiting } \\
\text { time }\end{array}$ & $\begin{array}{l}\text { No. negative } \\
\text { evaluations/No. of } \\
\text { responding clients } \\
\text { No. negative }\end{array}$ & $+63 \%$ & High \\
\hline Reliability & Lack of service & $\begin{array}{l}\text { evaluations/No. of } \\
\text { responding clients }\end{array}$ & $+46 \%$ & High \\
\hline Responsiveness & Average waiting time & Average waiting time & $+222 \%$ & Severe \\
\hline Credibility & $\begin{array}{l}\text { Negative comments on } \\
\text { media }\end{array}$ & $\begin{array}{l}\text { No. negative comments in } \\
\text { the period }\end{array}$ & $+84 \%$ & Average \\
\hline Security & $\begin{array}{l}\text { Negative comments on } \\
\text { media }\end{array}$ & $\begin{array}{l}\text { No. negative comments in } \\
\text { the period }\end{array}$ & $+818 \%$ & Severe \\
\hline
\end{tabular}

\subsection{Resilient Assessment}

In this section, we are going to analyze the effects of the incident on service evaluation in detail, and then put them in correlation with the elements of a resilient system. The aim is to validate the model proposed, by analyzing if, in relation to a low performance in terms of service evaluation, there had been a lack of commitment and investment in terms of resilience.

The analysis has been focused on evaluating the presence of organizational resilience elements and how these have been implemented in the case considered. For each of these elements, a judgement has been expressed on the following qualitative scale: inadequate, insufficient, sufficient, good, or excellent. 


\subsubsection{Resilience Indexes}

The organization implemented the procedures foreseen for emergency situations, adapting to the malfunction of the central system. The changes to normal procedures are a clear signal that something did not work at its best and therefore the organization's ability to adapt to the emergency and contingent situation was not optimal. The judgement on the adaptability of the organization was therefore insufficient. Furthermore, for what concerns reliability, the organization tried to guarantee the service by extending the time without interrupting it. Even if the inconveniences for customers were greater, in the end, the organization carried out a number of transactions that are comparable to normal periods of operation. For these reasons, the judgement on the reliability of the organization is sufficient.

The ability to respond to changes can be assessed by taking into consideration the overall assessment of the characteristics of the service. Looking at clients' evaluations of discomfort experienced, we can see how on average, the severity of inefficiencies had a considerable impact. For this reason, the organization's judgement for what concerns agility is inadequate.

The organization reached almost the same volume of transactions that it would have achieved in a normal period of operation. On this point; however, a relevant observation must be made, service times were extended and employees had to work beyond normal office hours to ensure an acceptable service level to clients. The judgement on effectiveness is sufficient, even if it is not possible to fully assess the achievement of the company's goals.

The term flexibility takes into account two aspects: the ability of an organization to cope with a variation in demand and the ability to offer different services with the same system without making numerous changes. One of the reasons that led to the malfunction was the increased workload. According to the organization, the central system had been sized to withstand a load similar to that experienced throughout the period of malfunctioning. Considering that the increase in workload was one of the possible causes of the malfunctioning and that the organization had not been able to provide all the services offered, the judgement on the flexibility of the organization is insufficient.

The organization managed to recover to the initial level. In fact, as mentioned above, the volume of transactions during the malfunction was comparable to the level of a normal operational day. This is possible because the organization devoted a considerable effort to restore an acceptable service level. Unfortunately, malfunctions had serious effects and repercussions even when the system was not experiencing problems, and also during the following days of restoration. Therefore, considering that the company could ensure a good recovery level in the first following days, the judgement expressed is therefore sufficient.

Looking at recovery time, the malfunction had a total duration of 7 hours over a whole working week. The recovery was followed by two milder periods of malfunction. On average, if we consider six days of work, the malfunction caused about an hour and ten minutes of inactivity per day. However, seven days of malfunctioning represent a fairly long period and therefore the assessment of the recovery time is insufficient.

All the evaluations on resilience characteristics are summarized in the following Table 4, where we highlighted the worst resilience dimensions impacting on the most severe service dimensions.

Many service features had a high impact on customers. From the average value line in Table 4, it can be seen how almost all the evaluations on the resilience indexes are lower than the sufficiency value. As pointed out in the previous sections, these resilience indexes express the effectiveness of resilience measures and characteristics on service dimensions and therefore on the judgement of clients. The malfunction experienced by customers resulted in a negative opinion about service evaluation, as confirmed by the analysis provided at the beginning of this section (paragraph 4.1). Indeed, this analysis shows that the severity of the impact is on average high for all service dimensions. If we combine this result with those emerging from the analysis of resilience, it can be said that there may be upstream. In particular, the average value of the resilience indexes is insufficient and therefore the quality of the service is affected. 
Table 4. Comparison between Resilience Indexes and Service Dimensions. Worst evaluations are highlighted.

\begin{tabular}{|c|c|c|c|c|c|c|c|}
\hline & & \multicolumn{6}{|c|}{ Service Dimensions } \\
\hline & & Competence & Access & Reliability & Responsiveness & Credibility & Security \\
\hline & Impact severity & Low & High & High & Severe & Average & Severe \\
\hline \multirow{7}{*}{$\begin{array}{l}\text { Resilience } \\
\text { dimensions }\end{array}$} & Adaptability & \multirow{7}{*}{$\begin{array}{l}\text { Sufficient } \\
\text { Insufficient }\end{array}$} & Insufficient & Insufficient & Insufficient & \multirow{3}{*}{ Sufficient } & Insufficient \\
\hline & Reliability & & Sufficient & Sufficient & Sufficient & & Sufficient \\
\hline & Agility & & Inadequat $\epsilon$ & Inadequate & Inadequate & & Inadequate \\
\hline & Effectiveness & & & Sufficient & Sufficient & \multirow{4}{*}{$\begin{array}{l}\text { Sufficient } \\
\text { Insufficient } \\
\text { Sufficient } \\
\text { Insufficient }\end{array}$} & Sufficient \\
\hline & Flexibility & & & & Insufficient & & Insufficient \\
\hline & Recovery Level & & & Sufficient & & & Sufficient \\
\hline & Recovery Time & & Insufficient & Insufficient & Insufficient & & \\
\hline
\end{tabular}

\subsubsection{Elements of Resilient Systems}

In this section, we want to analyze, in a backward perspective, how good the resilience measures the company put into action were. By crossing the evaluation of these measures with the average evaluations on resilience indexes (deriving from service evaluation), we can assess the meaningfulness of the framework proposed.

From the analysis of the company's history (derived from archival documents), a malfunction of these proportions was not expected, nor had the company ever faced something of this proportion before. The organization was not able to anticipate the problem, nor was it able to take the right precautions to avoid it. The new central system had been designed so as to be able to support very high workloads; however, a malfunctioning of this severity had not been taken into account. For this reason, the anticipation ability of the organization can be evaluated as insufficient.

The organization already experienced a similar malfunctioning a year before, albeit with a reduced scale and severity. During this period, part of the system had been modified, but still the organization itself did not know whether the two events could be connected to each other. From the organization's declarations, it emerges that following this past event, emergency procedures were not changed, just modified. The organization should be able to better learn from its mistakes and change the central system so that a similar problem will not happen again in the future. The judgement on the ability to learn from mistakes is insufficient.

The internal communication by the organization during the period of malfunctioning was carried out by means of e-mails, text messages, and phone calls. The communications, according to the operators of the organization, were imminent and sudden and all under the control of the service control room that monitored the system 24 hours a day and gave indications of what to do. The judgement on internal communication is therefore good.

Before the organization had a malfunction, there had been a series of minor episodes, which were hiding a bigger problem. According to company's statements, these episodes and warning signals had been underestimated. Greater attention would have allowed the organization to be ready to face the crisis. The company completely missed this point, and therefore the judgement can only be inadequate.

Most decisions made by the organization were based on emergency procedures. These procedures made it possible to limit a further escalation of the emergency. From statements made by the organization, it would seem that little had been done using improvisational capabilities. Nonetheless, according to different reports, several minor cases and crises within individual offices had been managed independently by employees, exhibiting non-negligible improvisational capabilities. For the above reasons, this aspect of organizational resilience culture can be evaluated as sufficient.

From the declarations released by the organization, it emerged that there was not a real system for continuous monitoring. In other words, the technical support company that managed the information system had its own control system, but it was not integrated with that of the organization. Furthermore, the two companies did not communicate the results of the analyses among themselves. When a problem occurred, the organization submitted it to the support company that solved it and did not 
provide any feedback to the organization. So, in this sense, there was no real central system to carry on a continuous monitoring effort. The organization managed its offices through the service control room, but that did not allow it to have an overall view of the system. The same statements indicate that there was a request from the organization to the assistance company to provide an integrated monitoring system. The evaluation of the continuous monitoring system is therefore insufficient.

The central system of the organization was composed of a primary system and a secondary system that replaces the first one in case of malfunction. Behind the malfunctions experienced; however, there was a misalignment of data between these two systems. In this case, the redundancy of the components did not help the organization to avoid the problem, but perhaps it could be one of the causes that determined its severity. The judgement on redundancy therefore suffers from the fact that in this case, it did not bring any benefit, resulting in an insufficient judgement.

Looking at the simulation processes, intended as system functionality tests, this aspect will encounter a negative judgement. Indeed, the new system was put into operation without being tested on a large scale, and in particular without testing a high load capacity. A more accurate test system and greater scenario simulation would have allowed the organization to mitigate the malfunction, furthermore it would have provided the company with more accurate emergency procedures. The judgement is therefore inadequate.

The organization, as mentioned above, was largely exposed to possible problems, failures, and malfunctions because the new system was put into operation without adequate tests. This is probably due to the greater importance given to commercial interests, instead of the system's functionality. Consequently, the judgement of the initial vulnerability state is sufficient, which actually expresses a considerable level of exposure for the company.

Table 5 summarizes the evaluations and compares them with average values of resilience indexes.

In Table 5 we highlighted the main causes that, according to the model, mostly affected negative performance in terms of resilience (expressed by the evaluations in the first line), which in turn affected service dimensions. We can see, for instance, how an insufficient performance in terms of anticipation ability affected the organizations' capabilities in terms of adaptability, flexibility, and recovery time. On the other hand, we can also state that the negative agility outcomes were mostly due to insufficient redundancy and inadequate simulation efforts. 
Table 5. Evaluation of the Elements of Resilient Systems and Resilience Indexes. Worst evaluations are highlighted.

\begin{tabular}{|c|c|c|c|c|c|c|c|c|}
\hline & & \multicolumn{7}{|c|}{ Resilience Dimensions } \\
\hline & & Adaptability & Reliability & Agility & Effectiveness & Flexibility & Recovery Level & Recovery Time \\
\hline & & Insufficient & Sufficient & Inadequate & Sufficient & Insufficient & Sufficient & Insufficient \\
\hline \multirow{8}{*}{$\begin{array}{c}\text { Resilience } \\
\text { characteristics }\end{array}$} & Anticipation ability & Insufficient & & & Insufficient & Insufficient & & Insufficient \\
\hline & Learning from mistakes & Sufficient & Sufficient & Sufficient & Sufficient & Sufficient & Sufficient & Sufficient \\
\hline & Internal communication & Good & Good & & Good & Good & Good & Good \\
\hline & Focus on minor aspects & Inadequate & Inadequate & & Inadequate & Inadequate & Inadequate & Inadequate \\
\hline & Improvisational capabilities & Sufficient & & Sufficient & Sufficient & \multirow{4}{*}{ Sufficient } & Sufficient & Sufficient \\
\hline & Continuous monitoring & Sufficient & Sufficient & Sufficient & Sufficient & & Sufficient & \\
\hline & Simulation & Inadequate & Inadequate & Inadequate & Inadequate & & Inadequate & \\
\hline & Initial vulnerability & Sufficient & Sufficient & Sufficient & Sufficient & & Sufficient & \\
\hline
\end{tabular}




\section{Conclusions}

Resilience is a key attribute for companies, vital for companies in order to secure business and its sustainability/continuity, while maintaining competitiveness. Some of the actions indicated to build resilience, like for instance continuous monitoring, improvisational capabilities, and anticipation ability can bring value in different business areas and functions, and not simply just contribute to building and developing resilience.

The model presented in this work has the aim of providing guidance from different points of view:

1. It gives indications and suggestions on which are the most interesting and valuable areas to focus on while configuring organizations as resilient systems.

2. It puts in correlation the concept of organizational resilience with service quality preservation, showing where companies should concentrate most to secure business and maintain competitiveness at the same time.

The model proposed can therefore be a useful tool in supporting resilience planning and actions of improvement. Furthermore, through the analysis of a real case, we provided evidence of how the model can be applied in a backward perspective to understand fault modes of specific events. In this way, it is possible to have a better understanding of incidents, their intrinsic dynamics, and seek for improvements Doing so, this model can become a resilience tool itself, contributing to learning from mistakes and in understanding minor events that might have been underestimated in the past.

However, the research has some limitations, especially the use of a single case for model testing and validating, and the use of what can be a large number of variables.

Future research works should adopt a wider variety of cases, in order to test and confirm the validity of the tool in different contexts and industries, to see whether its applicability is still effective, or if there are some contextual factors that might impose changes and loss of meaning to some aspects and variables.

Author Contributions: Conceptualization, A.A., B.C. and F.N.; methodology, A.A., B.C. and F.N.; validation, A.A., B.C. and F.N.; formal analysis, A.A., B.C. and F.N.; investigation, A.A., B.C. and F.N.; data curation, A.A., B.C. and F.N.; writing-original draft preparation, A.A., B.C. and F.N.; writing-review and editing, A.A., B.C. and F.N.; visualization, A.A., B.C. and F.N.; supervision, B.C. and F.N. All authors have read and agreed to the published version of the manuscript.

Funding: This research received no external funding.

Conflicts of Interest: The authors declare no conflict of interest.

\section{References}

1. Bhamra, R.; Dani, S.; Burnard, K. Resilience: The concept, a literature review and future directions. Int. J. Prod. Res. 2011, 49, 5375-5393. [CrossRef]

2. Branscomb, L.M. Sustainable cities: Safety and security. Technol. Soc. 2006, 28, 225-234. [CrossRef]

3. Holling, C.S. Resilience and Stability of Ecological Systems. Annu. Rev. Ecol. Syst. 1973, 4, 1-23. [CrossRef]

4. Fraccascia, L.; Giannoccaro, I.; Albino, V. Resilience of Complex Systems: State of the Art and Directions for Future Research. Complexity 2018, 2018, 1-44. [CrossRef]

5. Christopher, M.; Peck, H. Building the Resilient Supply Chain. Int. J. Logist. Manag. 2004, 15, 1-14. [CrossRef]

6. Hamel, G.; Välikangas, L. The quest for resilience. Harv. Bus. Rev. 2003, 81, 52-63.

7. Patriarca, R.; Di Gravio, G.; Costantino, F. Resilience engineering to assess risks for the air traffic management system: A new systemic method. Int. J. Reliab. Saf. 2016, 10, 323-345. [CrossRef]

8. Pettit, T.J.; Croxton, K.L.; Fiksel, J. Ensuring Supply Chain Resilience: Development and Implementation of an Assessment Tool. J. Bus. Logist. 2013, 34, 46-76. [CrossRef]

9. Patriarca, R.; Falegnami, A.; Costantino, F.; Bilotta, F. Resilience engineering for socio-technical risk analysis: Application in neuro-surgery. Reliab. Eng. Syst. Saf. 2018, 180, 321-335. [CrossRef]

10. Annarelli, A.; Nonino, F. Strategic and operational management of organizational resilience: Current state of research and future directions. Omega 2016, 62, 1-18. [CrossRef] 
11. Rose, A. Defining and measuring economic resilience to disasters. Disaster Prev. Manag. Int. J. 2004, 13, 307-314. [CrossRef]

12. Rose, A. Economic resilience to natural and man-made disasters: Multidisciplinary origins and contextual dimensions. Environ. Hazards 2007, 7, 383-398. [CrossRef]

13. Von Solms, R.; Van Niekerk, J. From information security to cyber security. Comput. Secur. 2013, 38, 97-102. [CrossRef]

14. Sheffi, Y.; Rice, J.B., Jr. A supply chain view of the resilient enterprise. MIT Sloan Manag. Rev. 2005, 47, 41-48.

15. Weick, K.E.; Sutcliffe, K.M. Managing the Unexpected: Assuring High Performance in an Age of Complexity; Jossey Bass Publishers: San Francisco, CA, USA, 2008.

16. Colicchia, C.; Dallari, F.; Melacini, M. Increasing supply chain resilience in a global sourcing context. Prod. Plan. Control. 2010, 21, 680-694. [CrossRef]

17. Schmitt, A.J.; Singh, M. A quantitative analysis of disruption risk in a multi-echelon supply chain. Int. J. Prod. Econ. 2012, 139, 22-32. [CrossRef]

18. Patriarca, R.; Costantino, F.; Di Gravio, G. Risk, safety, reliability and satellites: Chronicles of a fragmented research field. J. Space Saf. Eng. 2019, 6, 201-211. [CrossRef]

19. Choo, C.W. Organizational disasters: Why they happen and how they may be prevented. Manag. Decis. 2008, 46, 32-45. [CrossRef]

20. Turner, P.A.; Pidgeon, N.F. Man-Made Disasters, 2nd ed.; Butterworth-Heinemann: Oxford, UK, 1997.

21. Andersen, T.J. Information technology, strategic decision making approaches and organizational performance in different industrial settings. J. Strat. Inf. Syst. 2001, 10, 101-119. [CrossRef]

22. Welch, M.; Jackson, P.R. Rethinking internal communication: A stakeholder approach. Corp. Commun. Int. J. 2007, 12, 177-198. [CrossRef]

23. Elving, W.J. The role of communication in organisational change. Corp. Commun. Int. J. 2005, 10, 129-138. [CrossRef]

24. Aurand, T.W.; Gorchels, L.; Bishop, T.R. Human resource management's role in internal branding: An opportunity for cross-functional brand message synergy. J. Prod. Brand Manag. 2005, 14, 163-169. [CrossRef]

25. Punjaisri, K.; Evanschitzky, H.; Wilson, A. Internal branding: An enabler of employees' brand-supporting behaviours. J. Serv. Manag. 2009, 20, 209-226. [CrossRef]

26. Welch, M. The evolution of the employee engagement concept: Communication implications. Corp. Commun. Int. J. 2011, 16, 328-346. [CrossRef]

27. Turner, P.A. Man-Made Disasters; Wykeham: London, UK, 1978.

28. Westrum, R. Cultures with requisite imagination. In Verification and Validation of Complex Systems: Human Factor Issues; Wise, J.A., Hopkins, V.D., Stager, P., Eds.; Springer: Berlin, Germany, 1992; pp. 401-416.

29. Pavlou, P.A.; El Sawy, O.A. The "Third Hand": IT-Enabled Competitive Advantage in Turbulence Through Improvisational Capabilities. Inf. Syst. Res. 2010, 21, 443-471. [CrossRef]

30. Ates, A.; Bititci, U. Change process: A key enabler for building resilient SMEs. Int. J. Prod. Res. 2011, 49, 5601-5618. [CrossRef]

31. Bruneau, M.; Chang, S.E.; Eguchi, R.T.; Lee, G.C.; O’Rourke, T.D.; Reinhorn, A.M.; Shinozuka, M.; Tierney, K.; Wallace, W.A.; Von Winterfeldt, D. A Framework to Quantitatively Assess and Enhance the Seismic Resilience of Communities. Earthq. Spectra 2003, 19, 733-752. [CrossRef]

32. Fischer, K.; Hiermaier, S.; Riedel, W.; Häring, I. Morphology Dependent Assessment of Resilience for Urban Areas. Sustainability 2018, 10, 1800. [CrossRef]

33. Stevenson, M.; Spring, M. Flexibility from a supply chain perspective: Definition and review. Int. J. Oper. Prod. Manag. 2007, 27, 685-713. [CrossRef]

34. Helaakoski, H.; Iskanius, P.; Peltomaa, I. Agent-Based Architecture for Virtual Enterprises to Support Agility. In Establishing the Foundation of Collaborative Networks; Springer Science and Business Media LLC: Berlin, Germany, 2007; Volume 243, pp. 299-306.

35. Oerlemans, W.G.M.; Bakker, A.B. Burnout and daily recovery: A day reconstruction study. J. Occup. Heal. Psychol. 2014, 19, 303-314. [CrossRef]

36. McAllister, T. Research Needs for Developing a Risk-Informed Methodology for Community Resilience. J. Struct. Eng. 2016, 142, 4015008. [CrossRef]

37. Parasuraman, A.; Zeithaml, V.A.; Berry, L.J. A Conceptual Model of Service Quality and Its Implications for Future Research. J. Market. 1985, 49, 41-50. [CrossRef] 
38. Parasuraman, A.; Zeithaml, V.A.; Berry, L.J. Servqual: A Multiple-Item Scale for Measuring Consumer Perceptions of Service Quality. J. Retail. 1988, 64, 12-40.

39. Zeithaml, V.A. Defining and Relating Price, Perceived Quality, and Perceived Value; Report No. 87-101; Marketing Science Institute: Cambridge, MA, USA, 1987.

40. Garvin, D.A. Quality on the Line. Harv. Bus. Rev. 1983, 61, 65-73.

41. Hjorth-Anderson, C. The concept of Quality and the Efficiency of Markets for Consumer Products. J. Consum. Res. 1984, 11, 708-718. [CrossRef]

42. Olshavsky, R.W. Perceived Quality in Consumer Decision Making: An Integrated Theoretical Perspective. In Perceived Quality; Jacoby, J., Olson, J., Eds.; Lexington Book: Lexington, MA, USA, 1985.

43. Oliver, R. Measurement and Evaluation of Satisfaction Process in Retail Settings. J. Retail. 1981, 57, $25-48$.

44. Gronroos, C. Strategic Management and Marketing in the Service Sector; Chartwell-Bratt: London, UK, 1984.

45. Lehtinen, U.; Lehtinen, J.R. Service Quality: A Study of Quality Dimensions. Serv. Manag. Inst. 1982, 5, 25-32.

(C) 2020 by the authors. Licensee MDPI, Basel, Switzerland. This article is an open access article distributed under the terms and conditions of the Creative Commons Attribution (CC BY) license (http://creativecommons.org/licenses/by/4.0/). 